\title{
Fucoidan reduces the toxicities of chemotherapy for patients with unresectable advanced or recurrent colorectal cancer
}

\author{
MASAHIDE IKEGUCHI $^{1}$, MANABU YAMAMOTO ${ }^{1}$, YOSUKE ARAI $^{1}$, YOSHIHIKO MAETA $^{1}$, \\ KEIGO ASHIDA ${ }^{1}$, KUNIYUKI KATANO ${ }^{1}$, YASUNARI MIKI ${ }^{2}$ and TAKAYUKI KIMURA ${ }^{2}$ \\ ${ }^{1}$ Department of Surgery, Division of Surgical Oncology, Faculty of Medicine, Tottori University, \\ Yonago 683-8504; ${ }^{2}$ Marine Products Kimuraya, Co., Ltd., Sakaiminato 684-0072, Japan
}

Received September 27, 2010; Accepted January 4, 2011

DOI: $10.3892 / \mathrm{ol} .2011 .254$

\begin{abstract}
Combination chemotherapy with oxaliplatin plus 5-fluorouracil/leucovorin (FOLFOX) or irinotecan plus 5-fluorouracil/leucovorin (FOLFIRI) has become a standard regimen for advanced or recurrent colorectal cancer. Numerous studies have reported that long-term use of FOLFOX or FOLFIRI leads to better survival for these patients. Thus, control of the toxicity of these drugs may be crucial to prolonging survival. Fucoidan is one of the major sulfated polysaccharides of brown seaweeds and exhibits a wide range of biological activities. In the present study, we analyzed the effect of fucoidan on suppressing the toxicity of anti-cancer drugs. A total of 20 patients with unresectable advanced or recurrent colorectal cancer scheduled to undergo treatment with FOLFOX or FOLFIRI were randomly allocated into a fucoidan treatment group $(n=10)$ and a control group without fucoidan treatment $(n=10)$. Results showed that fucoidan regulated the occurrence of fatigue during chemotherapy. Chemotherapy with fucoidan was continued for a longer period than chemotherapy without fucoidan. Additionally, the survival of patients with fucoidan treatment was longer than that of patients without fucoidan, although the difference was not significant. Thus, fucoidan may enable the continuous administration of chemotherapeutic drugs for patients with unresectable advanced or recurrent colorectal cancer, and as a result, the prognosis of such patients is prolonged.
\end{abstract}

\section{Introduction}

To prolong the survival of patients with unresectable advanced or recurrent colorectal cancer, it is essential to continue effective chemotherapy for as long as possible. Since the introduction of oxaliplatin for use in Japan in April 2005, combination chemotherapy with oxaliplatin plus 5-fluorouracil (5-FU)/leucovorin

Correspondence to: Dr Masahide Ikeguchi, Department of Surgery, Division of Surgical Oncology, Faculty of Medicine, Tottori University, 36-1 Nishi-cho, Yonago 683-8504, Japan

E-mail:masaike@med.tottori-u.ac.jp

Key words: fucoidan, colorectal cancer, chemotherapy, fatigue
(LV) (FOLFOX) or irinotecan plus 5-FU/LV (FOLFIRI) has become the standard regimen for advanced or recurrent colorectal cancer, and a high response rate has been reported (1-3). However, FOLFOX and FOLFIRI are associated with severe toxicity, such as nausea, vomiting, stomatitis, diarrhea, fatigue, neutropenia, anemia, thrombocytopenia and liver dysfunction. A number of patients discontinue these effective chemotherapies due to toxicity. Thus, the prognosis of patients with unresectable advanced or recurrent colorectal cancer remains low despite advances in chemotherapeutic drugs.

To reduce the toxicity of chemotherapeutic drugs, various types of drugs or dietary supplements have been introduced (4-6). Among these supplements, fucoidan has been reported to exhibit anti-inflammatory, antiviral and anti-tumor activities (7-9). Fucoidan is a sulfated polysaccharide found mainly in various species of brown seaweeds such as kombu, wakame, mozuku and hijiki. Subsequently, fucoidan has become the focus of substantial pharmaceutical research.

The present study investigated whether fucoidan reduces the toxicity of chemotherapeutic drugs in patients with unresectable advanced or recurrent colorectal cancer.

\section{Materials and methods}

Patients. Between April 2008 and June 2009, 20 patients were diagnosed with unresectable advanced or recurrent colorectal cancer and were scheduled to undergo FOLFOX or FOLFIRI chemotherapy at our hospital. The Eastern Cooperative Oncology Group performance status of these patients was 0 or 1 , and they had adequate bone marrow (platelet count $\geq 100,000 / 1$, white blood cell count $\geq 4,000 / 1$, granulocyte count $\geq 1500 / 1$, hemoglobin level of $\geq 10.0 \mathrm{mg} / \mathrm{dl}$ ), renal (serum creatinine concentration $\leq 2.0 \mathrm{mg} / \mathrm{dl}$ ), and hepatic (serum bilirubin level $\leq 2.0 \mathrm{mg} / \mathrm{dl}$ ) functions. Adjuvant chemotherapy using 5-FU plus LV was administered to 9 of the 20 patients prior to enrollment in this study. The Ethics Committee of Tottori University approved treatment with fucoidan to reduce the toxicity of chemotherapeutic drugs in 2008 (approval no. 1223).

Informed consent was obtained from the 20 patients, who were randomly allocated to a fucoidan treatment group $(n=10)$ and a control group without fucoidan treatment $(n=10)$. The patients were followed up until July 2010. The patient details are shown in Table I. 
Table I. Patient characteristics.

$$
\text { + Fucoidan - Fucoidan P-value }
$$

\begin{tabular}{lccc}
\hline No. of patients & 10 & 10 & \\
Age (mean \pm SD, years) & $71.3 \pm 7.5$ & $69.6 \pm 8.8$ & 0.762 \\
Male/Female & $6 / 4$ & $7 / 3$ & 0.639 \\
ECOG & & & 0.653 \\
$\quad$ PS 0/1 & $5 / 5$ & $4 / 6$ & \\
Tumor & & & 0.653 \\
Primary/Recurrent & $4 / 6$ & $5 / 5$ & \\
Primary tumor & & & 0.639 \\
Colon/Rectum & $6 / 4$ & $7 / 3$ & \\
Previous chemotherapy & & & 0.653 \\
$\quad$ Yes/No & $4 / 6$ & $5 / 5$ & \\
Site of disease & & & 0.953 \\
Liver & 5 & 4 & \\
Lung & 2 & 2 & \\
Pelvis & 1 & 1 & \\
Peritoneum & 1 & 1 & \\
Lymph node & 1 & 1 & \\
Primary tumor & 0 & & \\
\hline
\end{tabular}

ECOG, The Eastern Cooperative Oncology Group; PS, performance status.

Chemotherapy. A number of versions of FOLFOX therapy exist, of which modified FOLFOX6 (mFOLFOX6) allows for more convenient administration and has been adopted by various medical institutions in association with popularization of outpatient chemotherapy. Thus, mFOLFOX6 has been the first-line therapy for patients with unresectable advanced or recurrent colorectal cancer at our hospital (10). A 2-h intravenous infusion of oxaliplatin $\left(85 \mathrm{mg} / \mathrm{m}^{2}\right)$ plus $1-\mathrm{LV}$ $\left(200 \mathrm{mg} / \mathrm{m}^{2}\right)$ was followed by a bolus intravenous injection of 5-FU $\left(400 \mathrm{mg} / \mathrm{m}^{2}\right)$, after which $5-\mathrm{FU}\left(2,400 \mathrm{mg} / \mathrm{m}^{2}\right)$ was administered by continuous infusion for $46 \mathrm{~h}$. However, 4 of the 20 patients requested FOLFIRI as first-line therapy. In the FOLFIRI regimen, on day $1,180 \mathrm{mg} / \mathrm{m}^{2}$ of irinotecan and $200 \mathrm{mg} / \mathrm{m}^{2}$ of 1-LV were administered as a 2-h infusion, prior to a $400 \mathrm{mg} / \mathrm{m}^{2}$ 5-FU intravenous bolus injection. Subsequently, $2,400 \mathrm{mg} / \mathrm{m}^{2}$ of $5-\mathrm{FU}$ was administered as a 46-h continuous infusion. The duration of one cycle of mFOLFOX6 was the same as that of FOLFIRI (2 weeks). Details of the chemotherapy regimens have been previously described (10).

Fucoidan treatment. Fucoidan is a sulfated polysaccharide that is extracted from brown seaweed, such as mozuku. In the present study, a high-molecular-weight product of fucoidan was used, which was derived from Cladosiphon okamuranus (Okinawamozuku) by Marine Products Kimuraya Co., Ltd. (Tottori, Japan). In the fucoidan group, each patient received $150 \mathrm{ml} /$ day of liquid that contained $4.05 \mathrm{~g}$ fucoidan for 6 months from the initial day of chemotherapy.
Table II. Major adverse events. ${ }^{a}$

$$
+ \text { Fucoidan - Fucoidan P-value }
$$

No. of patients $\quad 10 \quad 10$

Leukocytopenia

1

10

Neutropenia

3

0.305

Anemia

Thrombocytopenia

2

0.639

$0-0.53$

Nausea 1

0.136

Diarrhea 1

1.000

Stomatitis 3

Fatigue 1

Peripheral neuropathy 3

Liver dysfunction $\quad 0$

0.531

0.264

0.019

0.361

20.136

${ }^{a}$ Adverse events $\geq 2$.

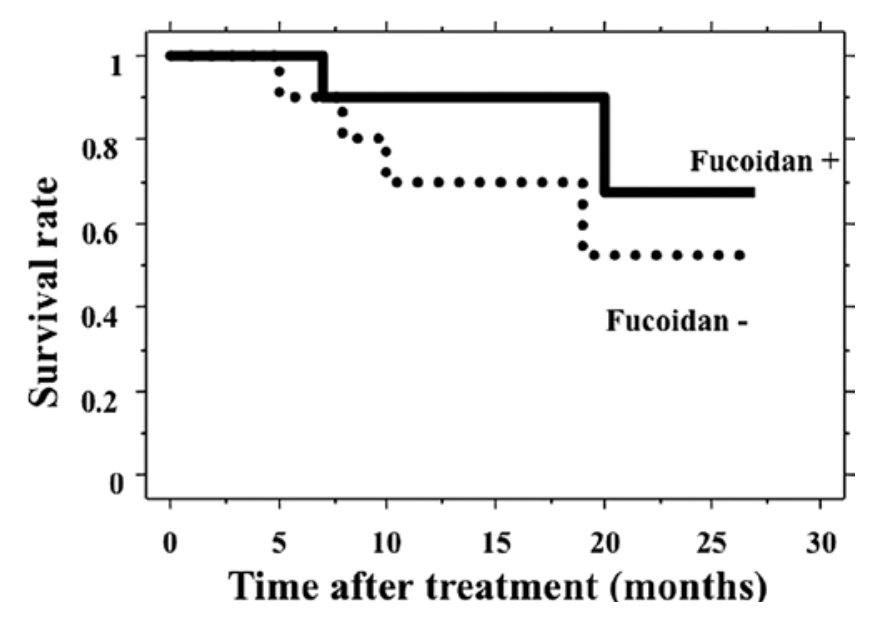

Figure 1. Survival curves of advanced or recurrent colorectal cancer patients. Solid line, survival curve of 10 patients who received fucoidan treatment. Dotted line, survival curve of 10 patients who did not receive fucoidan treatment. The difference was not significant $(\mathrm{P}=0.314)$.

Clinical assessment. All toxicities, with the exception of peripheral neuropathy, were graded according to the National Cancer Institute Common Toxicity Criteria (NCI CTC) (11). Peripheral neuropathy was graded according to the specific grading system (12). Hematological variables and clinical status were recorded every 2 weeks during the chemotherapy period. The drug dose level was reduced in the case of severe or persistent toxicity according to our protocol (10). In the case of persistent grade 3 toxicity or when grade 4 toxicity was recorded, chemotherapy was terminated.

Endpoints. The incidence and severity of adverse events were assessed as the primary endpoints, and patient survival, measured from the date of the first treatment until the patient succumbed to the disease, was assessed as the secondary endpoints.

Statistical analysis. The Chi-square test for independence, Fisher's exact probability test and the Mann-Whitney U test were used to compare patient characteristics, treatment status, 
adverse events and the anti-tumor effect. The survival rates of the two groups were estimated by the Kaplan-Meier method, and the statistical differences between survival curves were examined by the log-rank test. $\mathrm{P}<0.05$ was considered to be statistically significant.

\section{Results}

It was noted that fucoidan exhibited no side effects, such as allergic dermatitis. All 20 patients completed the 6 months of fucoidan therapy safely. Additionally, no patients succumbed due to chemotherapeutic toxicity. A total of 307 cycles of mFOLFOX6 or FOLFIRI were administered during the study, with a median of 15.4 cycles per patient (range 7-38). The average number of treatment cycles (19.9) in the fucoidan group was significantly greater than that in the control group (10.8 cycles, $\mathrm{P}=0.016$ ).

The observed toxicities of the chemotherapeutic drugs are listed in Table II. No patients presented with severe toxicity (grade 4) in either group. The occurrences of diarrhea and neurotoxicity were not suppressed by fucoidan. Myelosuppression was found to be similar in the fucoidan and control groups. In contrast, general fatigue was detected in $60 \%$ of the control group, but was significantly suppressed to $10 \%$ in the fucoidan group (Table II).

Patients were followed up at our hospital. The median follow-up period of the 20 patients was 15 months (range 5-27). During the follow-up period, 6 patients (2 in the fucoidan group and 4 in the control group) succumbed due to colorectal cancer progression. The survival of the 10 patients receiving fucoidan treatment was longer than that of the 10 patients in the control group, but the difference was not significant ( $\mathrm{P}=0.314$, Fig. 1$)$.

\section{Discussion}

Fucoidan is one of the major sulfated polysaccharides of brown seaweeds, and it has a wide range of biological activities. Choi et al (13) found that fucoidan protects gastric mucosa from inflammatory cytokine-mediated oxidative damage in rats. Hayashi et al (7) reported that fucoidan reduces $\mathrm{CCl}_{4}$ induced acute and chronic liver failure with hepatic fibrosis. The anti-inflammatory activity of fucoidan was demonstrated in rats (14), and fucoidan conferred no toxicity in rats at high doses (15). Thus, fucoidan is anticipated to improve human health, and has been widely distributed as a foodstuff but not as a drug. However, the detailed mechanism of action of fucoidan remains to be verified, and its effects in humans have yet to be determined.

In the present study, we analyzed whether fucoidan protects patients from the toxicity of anti-cancer drugs. Nausea, vomiting, diarrhea, general fatigue and bone marrow suppression are well-known common adverse effects of anticancer drugs. Peripheral neuropathy is specific for oxaliplatin. We found that fucoidan suppressed the occurrence of general fatigue in colorectal cancer patients during chemotherapy. It has been demonstrated that fatigue reduces the individual resources of patients, affects their nutritional status, increases morbidity and can have a negative impact on the dose intensity of cancer therapy (16). Iop et al (16) reported that fatigue, which was graded using NCI CTC, was detected in almost $30 \%$ of patients receiving chemotherapy. In the present study, grade 2 and 3 fatigue was detected in $60 \%$ of colorectal cancer patients during chemotherapy. The use of antidepressants may also play a role in the treatment of fatigue, and a number of patients are administered chemical supplements of unproven efficacy. However, no published data exist to confirm this hypothesis. In our study, patients who received fucoidan were able to endure prolonged chemotherapy without fatigue. However, fucoidan did not have an impact on other adverse effects of anti-cancer drugs. The mechanisms that explain chemotherapy-induced fatigue remain to be determined, and no general treatment is currently available to alleviate the symptoms.

Fucoidan has also been found to play a significant role in tumor suppression (17-20). Yamasaki-Miyamoto et al (8) and Hyun et al (21) showed that fucoidan activates caspase- 8 or extracellular signal-regulated kinase and induces apoptosis in tumor cells. These pro-apoptotic effects of fucoidan have not been detected in normal cells. However, no indisputable evidence exists that fucoidan prolongs the survival of cancer patients, even in animal models with human tumor implants. In the present study, although the number of patients was limited and the results were not statistically significant, the prognosis of patients with unresectable advanced or recurrent colorectal cancer was more favorable upon treatment with fucoidan than without. This may be explained by the fact that fucoidan prolonged the duration of the chemotherapy by suppressing the toxicity of the anti-cancer drugs or through an anti-cancer effect of fucoidan itself. Therefore, large controlled studies are required to evaluate the therapeutic effect of fucoidan for unresectable advanced or recurrent colorectal cancer.

\section{References}

1. Goldgerg RM, Sargent DJ, Morton RF, et al: A randomized controlled trial of fluorouracil plus leucovorin, irinotecan, and oxaliplatin combinations in patients with previously untreated metastatic colorectal cancer. J Clin Oncol 22: 23-30, 2004.

2. Tournigand $\mathrm{C}$, André $\mathrm{T}$, Achille E, et al: FOLFIRI followed by FOLFOX6 or the reverse sequence in advanced colorectal cancer: a randomized GER-COR study. J Clin Oncol 22: 229-237, 2004.

3. Japanese Society for Cancer of the Colon and Rectum: Guidelines for Management of Colon Cancer (for Physicians, version 2005) Kanehara \& Co., Ltd., Tokyo, 2005.

4. Lee CK, Park KK, Hwang JK, Lee SK and Chung WY: Extract of Prunus persica flesh (PPFE) improves chemotherapeutic efficacy and protects against nephrotoxicity in cisplatin-treated mice. Phytother Res 23: 999-1005, 2009.

5. Block KI, Koch AC, Mead MN, Tothy PK, Newman RA and Gyllenhaal C: Impact of antioxidant supplementation on chemotherapeutic toxicity: A systematic review of the evidence from randomized controlled trials. Int J Cancer 123: 1227-1239, 2008.

6. Eussen S, Klungel O, Garssen J, et al: Support of drug therapy using functional foods and dietary supplements: focus on statin therapy. Br J Nutr 103: 1260-1277, 2010.

7. Hayashi S, Itoh A, Isoda $\mathrm{K}$, Kondoh M, Kawase $\mathrm{M}$ and Yagi K: Fucoidan partly prevents $\mathrm{CCl}_{4}$-induced liver fibrosis. Eur J Pharmacol 580: 380-384, 2008.

8. Yamasaki-Miyamoto $\mathrm{Y}$, Yamasaki $\mathrm{M}$, Tachibana $\mathrm{H}$ and Yamada K: Fucoidan induces apoptosis through activation of caspase-8 on human breast cancer MCF-7 cells. J Agric Food Chem 57: 8677-8682, 2009.

9. Saitoh Y, Nagai Y and Miwa N: Fucoidan-Vitamin C complex suppresses tumor invasion through the basement membrane, with scarce injuries to normal or tumor cells, via decreases in oxidative stress and matrix metalloproteinases. Int J Oncol 35: 1183-1189, 2009. 
10. Sugimoto S, Katano K, Kanazawa A, et al: Multicenter safety study of mFOLFOX6 for unresectable advanced/recurrent colorectal cancer in elderly patients. J Exp Clin Cancer Res 28 109, 2009.

11. Therasse P, Arbuck SG, Eisenhauer E, et al: New guidelines to evaluate the response to treatment in solid tumors. J Natl Cancer Inst 92: 205-216, 2000.

12. Lévi F, Misset JL, Brienza S, et al: A chronopharmacologic phase II clinical trial with 5-fluorouracil, folinic acid, and oxaliplatin using an ambulatory multichannel programmable pump. High antitumor effectiveness against metastatic colorectal cancer. Cancer 69: 893-900, 1992.

13. Choi JI, Raghavendran HRB, Sung NY, et al: Effect of fucoidan on aspirin-induced stomach ulceration in rats. Chem Biol Interact 183: 249-254, 2010.

14. Del Bigio MR, Yan HJ, Campbell TM and Peeling J: Effect of fucoidan treatment on collagenase-induced intracerebral hemorrhage in rats. Neurol Res 21: 415-419, 1999.

15. Kim KJ, Lee OH, Lee HH and Lee BY: A 4-week repeated oral dose toxicity study of fucoidan from the Sporophyll of Undaria pinnatifida in Sprague-Dawley rats. Toxicology 267: 154-158, 2010.
16. Iop A, Manfredi AM and Bonura S: Fatigue in cancer patients receiving chemotherapy: an analysis of published studies. Ann Oncol 15: 712-720, 2004

17. Riou D, Colliec-Jouault S, Pinczon du Sel D, et al: Antitumor and antiproliferative effects of a fucan extracted from Ascophyllum nodosum against a non-small-cell bronchopulmonary carcinoma line. Anticancer Res 16: 1213-1218, 1996.

18. Itoh $\mathrm{H}$, Noda $\mathrm{H}$, Amano $\mathrm{H}$, Zhuaug $\mathrm{C}$, Mizuno $\mathrm{T}$ and Ito $\mathrm{H}$ : Antitumor activity and immunological properties of marine algal polysaccharides, especially fucoidan, prepared from Sargassum thunbergii of Phaeophyceae. Anticancer Res 13: 2045-2052, 1993.

19. Maruyama $H$, Tamauchi $H$, Hashimoto $M$ and Nakano $T$ : Antitumor activity and immune response of Mekabu fucoidan extracted from Sporophyll of Undaria pinnatifida. In Vivo 17: 245-249, 2003.

20. Koyanagi S, Tanigawa N, Nakagawa H, Soeda S and Shimeno H: Oversulfation of fucoidan enhances its anti-angiogenic and antitumor activities. Biochem Pharmacol 65: 173-179, 2003.

21. Hyun JH, Kim SC, Kang JI, et al: Apoptosis inducing activity of fucoidan in HCT-15 colon carcinoma cells. Biol Pharm Bull 32: 1760-1764, 2009. 